Anastasia Drackert (Bochum), Wolfgang Stadler (Innsbruck)

\title{
Leistungsbeurteilungskompetenz stärken - Von der Theorie zur Praxis am Beispiel eines Lehrszenarios zur Verbesserung von Hörverstehensaufgaben im Russischunterricht
}

In research, listening comprehension is considered to be one of the most complex skills, challenging teachers of Russian not only when teaching but also when evaluating their students' performances. Therefore, it is not surprising that the listening skill is only tested and assessed "sometimes" (cf. Drackert \& Stadler 2017) in the Russian language classroom. This article presents a short overview of the theory of listening comprehension and introduces a teaching scenario that can be used in subject-specific language courses both pre-service and in-service, hereby developing and strengthening the language assessment literacy of teachers of Russian as a foreign language.

Аудирование, которое в дидактической литературе считается одним из самых трудных видов речевой деятельности, по-прежнему представляет особую сложность для преподавателей русского языка не только при обучении, но и при оценке знаний учащихся. Поэтому тот факт, что учителя только «иногда» тестируют и оценивают этот навык на уроках русского языка (см. Drackert \& Stadler 2017), совсем не удивляет. В данной статье представлен обзор теории аудирования, а также учебный сценарий, который может быть использован на семинарах по дидактике в университете, в педагогической практике или на курсах повышения квалификации преподавателей с целью улучшения их компетенции в области тестирования. 


\section{Einleitung}

„Lesen und Hören ist immer das Problem des Materials, wo kriege ich jetzt was her, das passt. Also wir haben /die Franzosen, Engländer sowieso, Spanier haben Hefte voll mit Übungen und in Russisch, wir haben /jeder erstellt irgendwie seine eigenen $\mathrm{Sa}$ chen, dann passen die halt speziell auf diese Klasse, aber auf meine nicht, weil da dieser Wortschatz noch nicht drinnen ist und darum also Hören, Lesen finde ich sehr schwierig."

(Persönliches Interview [3] mit einer Russischlehrerin aus Wien, Russisch als 2. Lebende Fremdsprache, Sekundarstufe II, 28.08.2017, 16:48-17:17).

Hören als primäre rezeptive Fertigkeit ist eine jener sprachlichen Aktivitäten, die Heyer (2014: 108) als ,unverzichtbaren Bestandteil des Erlernens einer Lautsprache" - und somit auch der russischen Sprache bezeichnet. Ott (1995: 518) spricht beim Hörverstehen von der „schwierigsten kognitiven Leistung im Fremdsprachenunterricht“ - einer Leistung, die sowohl im Unterricht als auch bei der Kompetenzüberprüfung Lehrenden und Lernenden Schwierigkeiten macht (vgl. z.B. Sherrow 1971). Die Gründe dafür liegen nicht nur in der kognitiven und sozialen Dimension der Fertigkeit Hören (vgl. Goh \& Vandergrift 2009) bzw. in der Frage, ob Hören im Unterricht bzw. in der Testsituation interaktional oder transaktional aufzufassen sei (Guan \& Jin 2010), sondern auch in der Fokussierung auf Hören als prozessorientierte Kompetenz oder als produktorientierte Performanz (Abbott \& Nguyen 2016). Sowohl das eine (Hören als Prozess) als auch das andere (Hören als Produkt) erfordern unterschiedliche Hörstile bzw. -strategien, die entwickelt, geschult und trainiert werden müssen. Walker (2014: 173) bezeichnet Hörverstehen als ,the most difficult skill to teach“; Hören ist 
aber sicherlich auch eine der am schwierigsten zu überprüfenden Fertigkeiten im Fremdsprachenunterricht (vgl. Kranert 2013).

In einer Untersuchung zur Leistungsbeurteilungskompetenz (Drackert \& Stadler 2017), durchgeführt mit 198 Befragten in Deutschland, Österreich, der Schweiz und Südtirol zwischen 11/2016 und 02/2017, geben Russischlehrkräfte zwar an, die Fertigkeit Hören „manchmal“ zu überprüfen $\left[M W=3,17\right.$ auf einer Skala von 1 (,nie') bis $\left.4\left(, \mathrm{oft}^{\circ}\right)\right]$, und sie rangiert gemeinsam mit den anderen Fertigkeiten Lesen, Schreiben und Sprechen unter den Fortbildungswünschen von RussischlehrerInnen an erster Stelle [25,6\% der Russischlehrerkräfte in Österreich, Deutschland, der Schweiz und Südtirol machten diese Angabe]. Doch sagen diese Zahlen nichts darüber aus, wie kompetent diese Fertigkeit unterrichtet bzw. wie kompetent sie überprüft wird. Zudem ist Hören jene Fertigkeit unter den vier Skills, die eben „,manchmal“, aber seltener als Sprechen, Schreiben oder Lesen überprüft bzw. getestet wird.

Der vorliegende Beitrag widmet sich deshalb der komplexen Fertigkeit Hören, deren Verständnis in den letzten Jahren zweifellos Veränderungen erfahren hat (vgl. Osada 2004; Grotjahn 2012) - sei es bei der unterschiedlichen Gestaltung interaktiver Hörverstehensaufgaben (HVA) in aktuellen Russischlehrwerken im Vergleich zu früheren, als Hören lediglich Zuhören oder „passives Mitlesen“ bei offenem Lehrbuch war (vgl. Balas, Kaltseis \& Schmidt 2017), oder als diese Fertigkeit im Jahr 2014 in die teilstandardisierte Reife- und Diplomprüfung in Österreich integriert wurde (vgl. Hinger \& Stadler 2018) bzw. jüngst, als diese Fertigkeit Eingang in die Abiturprüfungen in Deutschland fand (vgl. Vorabhinweise zum Abitur 2018) ${ }^{1}$.

\footnotetext{
${ }^{1}$ https://tinyurl.com/y9docnsu

Anastasia Drackert und Katrin Bente Karl (Hg.), Didaktik der slawischen Sprachen

Beiträge zum 2. Arbeitskreis in Innsbruck (19.02.-20.02.2018)

(C) 2019 innsbruck university press, ISBN 978-3-903187-80-1, DOI 10.15203/3187-80-1
} 
Im Folgenden soll gezeigt werden, wie die Leistungsbeurteilungskompetenz von RussischlehrerInnen (pre-service, in-service) im Bereich „Hörverstehen überprüfen“ verbessert werden kann, denn „Beobachtungen in der Praxis haben ergeben, dass Lehrkräfte sich entweder auf in Lehrwerken vorgegebene Materialien beschränken“ (Heyer 2010: 4) oder ,selbst Hörtexte entwickeln, um Defizite an geeigneten Materialien auszugleichen“"(Heyer 2014: 119). Mit der bloßen Auswahl oder der selbstständigen Entwicklung eines Hörtextes ist es allerdings nicht getan. Anhand konkreter Beispiele von HVA werden Richtlinien und Rahmenbedingungen präsentiert, die als Checkliste für die Erstellung weiterer rezeptiver Testaufgaben verwendet werden können. Durch das Bewusstmachen der einzelnen theoriegeleiteten und praxisorientierten Schritte werden bei der Aufgabenerstellung die Herausforderungen und Schwierigkeiten von kompetenzorientierten Klassenarbeiten bzw. Prüfungsaufgaben erläutert.

Ziel des Beitrags ist es, ein Lehr- bzw. Fortbildungsszenario zu präsentieren, das Lehrkräften an der Schule und der Universität, Fach- und AGLeiterInnen sowie FortbildnerInnen verdeutlicht, wie sie die Auswahl der Texte und die Verwendung der Prüfungssaufgaben rechtfertigen können und wie sie zu reliablen und validen Interpretationen der Ergebnisse in der Leistungsüberprüfung kommen und dadurch ihre eigene Bewertungskompetenz stärken.

\section{Theoretischer Hintergrund}

Es scheint für den Zweck dieses Beitrages zielführend, einen komprimierten Überblick über den Forschungsstand der letzten Jahre zur Exploration des Hörverstehens zu geben. Um trotz der Kürze des Beitrags dennoch die Komplexität und Variabilität dieses Prozesses aufzuzeigen, genügt es, auf einige Begriffe zu verweisen, wie Hören z.B. in der deutsch-, englisch- 
oder russischsprachigen Forschungsliteratur bezeichnet wird: schwierige Sprachtätigkeit (Heyer 2010), zielgerichtete Aktivität (Grotjahn, Porsch \& Tesch 2010), komplexer Prozess (Thaler 2009); (increasingly) vital skill (Sherrow 1971; Iskold 2008), complex skill (Chastain 1979; Abbott \& Nguyen 2016), important and distinctive skill (Rubin \& Thompson 1996), highly integrative skill (Vandergrift 1999), important skill (Goh \& Vandergrift \& 2009), crucial skill (Liakhnovitch, Masalimova \& Porchesku 2014), the most difficult skill (Walker 2014); очень важное умение (AverkoAntonovič 2013), один из наиболее сложных [...] видов речевой деятельности (Anisimova 2015; Volosova 2017).

Dennoch ist die Fertigkeit Hören in der Fachliteratur bis heute ein Cinderella Skill (Nunan 1997) geblieben, die im Fremdsprachenunterricht wie auch Osada (2004: 57) festhält - nach wie vor ,etwas vernachlässigt und schlecht unterrichtet [wird]" und einer der ,am wenigsten verstandenen Prozesse“ ist. Dies gilt auch für die Forschung, da (empirische) Studien zur Erforschung des Hörverstehens wesentlich seltener sind als jene zur rezeptiven Fertigkeit Lesen (vgl. Nold \& Rossa 2007). Auch im Russischunterricht kommt der Bewertung von Leistungen im Bereich des Hörverstehens zu wenig Bedeutung zu (vgl. Drackert \& Stadler 2017), denn viele Lehrpersonen betrachten Hören nach wie vor als eine ,ppassive Fertigkeit" (Anderson \& Lynch 1988), die sich im Idealfall von selbst bzw. „osmotisch“ entwickelt (vgl. z.B. Osada 2004: 54). Lernende zeigen gerade dieser Fertigkeit gegenüber Ängste (vgl. Conti 2015), was vor allem auf Schwierigkeiten wie das nicht kontrollierbare Sprechtempo in Echtzeit oder auf phonetisch-phonologisch-prosodische (z.B. Markierung von Wortgrenzen, Elision, Intonation etc.) und lexikalisch-grammatikalische Besonderheiten mündlicher Sprache (wie umgangssprachlicher Wortschatz, Wortstellung, Segmentierung) bei der ephemeren auralen Rezeption zurückzuführen ist. 
Um einen Einblick zu bekommen, dass Hören nicht länger als „,passive“, sondern als komplexe, (inter-)aktive und kommunikative Fertigkeit zu gelten hat, können Lehrende auf verschiedene Hörverstehensmodelle (wie z.B. Field 2011; Khalifa \& Weir 2009) zurückgreifen. Diese Modelle verknüpfen die unterschiedlichen kognitiven Prozesse miteinander und zeigen, wie aus Gehörtem gleichzeitig und parallel auf mehreren Ebenen Bedeutung generiert wird. Als Standardwerke für den Unterricht und die Leistungsbeurteilung von Hörverstehen gelten weiterhin Fields Listening in the Language Classroom (2008) bzw. Bucks Assessing Listening (2001), wobei Letzteres in das Testen und Bewerten von Hörverstehensprozessen einführt und Kapitel abdeckt, die sich u. a. den spezifischen Besonderheiten des Hörverstehens, der Definition des Konstrukts, der Erstellung von Aufgaben und der Auswahl geeigneter Texte widmen. Eine Gegenüberstellung zwischen Hörverstehen und Hör-Sehverstehen bieten Grotjahn, Porsch \& Tesch (2010); Grotjahn (2012) gewährt Einblicke in das Konstrukt und die Messung von Hörverstehensleistungen. Viele der Arbeiten thematisieren das Hören in der ersten lebenden Fremdsprache (z.B. Boge 2012, Nold \& Rossa 2007) oder in der Zweitsprache Englisch. Diese Arbeiten können aber durchaus auch als Anregung für ,Russisch als Fremdsprache“" gesehen werden, wofür erwartungsgemäß nur wenig Publikationen vorhanden sind (z.B. Bauer 1986; Heyer 2014) - sieht man von kleineren Beiträgen (Heyer 2010; Fitzer 2014; Fischer 2015), Materialsammlungen des ThILLM (2006) ${ }^{2}$ oder von Fortbildungspräsentationen wie jener von Birzer \& Steinbach $(2015)^{3}$ ab. Manche der praxisorientierten Veröffentlichungen gehen auf Herausforderungen ein, die Lernende auf der Stufe der elementaren Sprachverwendung erleben (Heyer 2010) oder sie schlagen verschiedene Phasen der Erarbeitung und Sicherung bei der Hörverstehensentwicklung und -überprüfung vor (Fitzer 2014). Eine Sammlung von (nicht pilotierten bzw. feldgetesteten) Hörtexten und -aufgaben für das Russische (B1 und B2) bieten z.B. Bauer \& Kolesnik-Eigentler (2013).

\footnotetext{
${ }^{2}$ https://tinyurl.com/y83e3e3u

${ }^{3}$ https://tinyurl.com/ybq8v5j7
} 
Den meisten Definitionen von Hörverstehen in der Fachliteratur ist gemeinsam, dass sprachliche Äußerungen dekodiert, interpretiert und reflektiert werden müssen (vgl. z.B. Nold \& Rossa 2007: 178), wobei verschiedene Textgenres, Themen und kommunikative Kontexte zu berücksichtigen sind. Die unterschiedlichen Hörverstehensmodelle unterstreichen neben der sprachlichen und der strategischen Kompetenzebene auch die Rolle des Weltwissens, die unumgänglich ist, um aus dem Gehörten Bedeutung(en) leichter entschlüsseln zu können, diese Bedeutungen miteinander zu verknüpfen und im Kurz- oder Langzeitgedächtnis zu speichern. Zwei Typen von Verstehensprozessen (bottom up und top down) werden für die Sinnkonstruktion gehörter Texte vorausgesetzt und in der Forschungsliteratur ausführlich besprochen, wobei ersterer aufsteigend und datengesteuert ist und sprachliches Wissen verarbeitet, letzterer absteigend und wissensgesteuert abläuft und nicht-sprachliches Vor- und Weltwissen (wie Erwartungen und Schemata) zur Bedeutungserschließung nutzt (vgl. z.B. Grotjahn 2012: 75). Ebenso wird in der wissenschaftlichen Forschung der Frage nachgegangen, ob Hörtexte didaktisiert oder authentisch, schriftlich oder mündlich, kurz oder lang sein sollen, ob sie einmal oder mehrmals präsentiert werden sollen, wie sehr sie an die Erfordernisse der lernersprachlichen Entwicklung (Nold \& Rossa 2007: 182) angepasst sein sollen bzw. ob sie an diese überhaupt angepasst werden können. Bei der zentralen Verarbeitung von Hörtexten spielen metakognitive Komponenten und Wissenskomponenten eine wesentliche Rolle: Warum hören wir Texte? Welches Wissen hilft uns, Texte besser zu verstehen? Welche Rolle nehmen wir als HörerIn ein? Sind wir z.B. lediglich MithörerIn bei einem Gespräch, sind wir Radio- oder PodcasthörerIn oder aktive/r GesprächsteilnehmerIn, die zwischen Sprecher- und der Hörerrolle wechseln? HVA hätten somit ein sehr breites Konstrukt abzudecken, das in der gesamten Fülle bzw. in Abhängigkeit des jeweiligen Definitionsansatzes schwer zu fassen ist.

Anastasia Drackert und Katrin Bente Karl (Hg.), Didaktik der slawischen Sprachen

Beiträge zum 2. Arbeitskreis in Innsbruck (19.02.-20.02.2018)

(C) 2019 innsbruck university press, ISBN 978-3-903187-80-1, DOI 10.15203/3187-80-1 
Grotjahn (2012: 73) spricht von einem kompetenz-, einem aufgabenbasierten und einem interaktiven Ansatz der Konstruktdefinition. Eine Schwierigkeit sieht auch er darin, dass jene Prozesse, die die Hörverstehenskompetenz ausmachen, nicht beobachtet werden können. Aufgaben, die bei der Schulung oder der Überprüfung des Hörverständnisses gestellt werden, sollen unterschiedliche Performanzen evozieren, die jenen in zielsprachlichen Kommunikationssituationen ähnlich und mit der Lösung zielsprachenrelevanter Aufgaben kompatibel sind. Auf den Punkt gebracht heißt das: Welche Aufgabe ist ein valider Indikator für kompetentes Hörverstehen? Da verschiedene Faktoren wie die Zahl der SprecherInnen, die Sprechgeschwindigkeit, die Unterscheidbarkeit der Stimmen, der Akzent, Dialekt, die Intonation, die Artikulation, das Gesprächsthema etc. den Schwierigkeitsgrad der Aufgabe wesentlich beeinflussen (vgl. z.B. Anisimova 2015), muss den Merkmalen von Aufgaben eine besondere Aufmerksamkeit zuteil werden. Zur Schulung des Hörverstehens werden üblicherweise drei Phasen eingesetzt, die Übungen bzw. Aufgaben vor, während und nach dem Hören vorsehen (Thaler 2009).

Buck (2001) empfiehlt auf Grund der aufgezeigten Komplexität des Hörens, ein default-Konstrukt (vgl. Vandergrift \& Goh 2009: 405) ins Auge zu fassen, das für das sichere Lösen jedweder HVA konstituierend ist (vgl. Nold \& Rossa 2007: 184):

- Verstehen authentischer, gesprochener Sprache;

- Entschlüsseln der notwendigen Verstehensprozesse (necessary information) und

- Ziehen von eindeutigen Schlussfolgerungen.

Da der Russischunterricht zunehmend handlungs- und kompetenzorientiert gestaltet ist und den Empfehlungen des GeR (Europarat 2001) für einen kommunikativen Sprachunterricht folgt, stehen Hörintentionen wie globales, selektives und detailliertes Hören im Mittelpunkt von HVA. 


\section{Das Konstrukt von Hörverstehensaufgaben (HVA)}

Der GeR (vgl. Europarat 2001: 214; Council of Europe 2018: 55) legt für das Hörverstehen fünf Beispielskalen vor:

- Hörverstehen allgemein

- Gespräche zwischen Muttersprachlern verstehen

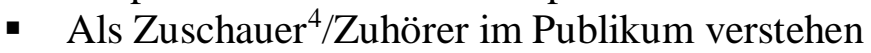

- Ankündigungen, Durchsagen und Anweisungen verstehen

- Radiosendungen und Tonaufnahmen sowie Fernsehsendungen und Filme verstehen

Eine weitere Beispielskala aus dem Bereich der kommunikativen Strategien ist Hinweise identifizieren und erschließen, die für die Schulung und Überprüfung der rezeptiven Fertigkeit Hören ebenfalls zu berücksichtigen ist. Diese Zusammenstellung von Skalen zeigt, dass transaktionales, nicht interaktionales Hören im Vordergrund des GeRKonstrukts steht. Die Forderung nach authentischer Sprache mit typischen Merkmalen von Mündlichkeit scheint daher nicht in jedem Fall bedingungslos umsetzpflichtig zu sein. Auch bei den „Deutsch-EnglischSchülerleistungen-International“ (DESI) kommen Texte zum Einsatz, denen scripted texts als Grundlage dienen und die schriftsprachlichen Konventionen folgen (vgl. Nold \& Rossa 2007: 182; Grotjahn 2012: 82). Die Hörtexte, die bei einer Klassenarbeit, einer Klausur oder einer Prüfung eingesetzt werden, müssen jedenfalls den Texten ähnlich sein, die Lernende aus dem Unterricht kennen. Eine Mischung von Text(sort)en mit und ohne Bezug zur (authentischen) Verwendung im kommunikativen Russischunterricht ist durchaus empfehlenswert. Auf alle Fälle sollen „kontextualisierte Beispiele für die zu lehrenden [- bzw. die gelehrten und

\footnotetext{
${ }^{4}$ Hör-/Sehverstehen bleibt in diesem Beitrag ausgeklammert.
} 
zu überprüfenden -] sprachlichen Inhalte“ (GeR Europarat 2001: 143) zur Verfügung stehen.

Klausuren an der Schule erheben nicht nur den Sprachstand, der an die Vermittlung gewisser (sprachlicher und thematischer) Inhalte und ausgewählter Kommunikationsziele der Unterrichtseinheiten gebunden ist, sondern sie evaluieren ihn auch. Für das B1-Niveau wird z.B. ein Spektrum an sprachlichen Mitteln gefordert, das ,,den meisten Themen, die zum Alltagsleben gehören“, eigen ist: Familie, Hobbys und Interessen, Arbeit, Reisen, aktuelle Ereignisse (vgl. GeR Europarat 2001: 216). Der Inhalt ist weniger „Voraussagbar“ als bei A2-Themen; es können Erfahrungen beschrieben und ein Informationsaustausch geboten werden, wie z.B. jene, die bei einer detaillierten Wegbeschreibung oder einer (kurzen) Erzählung bewältigt werden müssen. Größere Mengen unkomplizierter Sachinformationen (aus vertrauten Themen) bieten sich ebenfalls als inhaltliche Muster für kommunikative Situationsthemen auf B1-Niveau an (vgl. GeR Europarat 2001: 216). Die Artikulation hat klar, der Akzent vertraut, das Tempo eher langsam zu sein und es muss Standardsprache gesprochen werden. Den Rezipienten obliegt es, sowohl Hauptaussagen (general messages) und Hauptpunkte (main points) als auch Einzelinformationen (specific details) verstehen zu können (vgl. GeR Europarat 2001: 72; CEFR Council of Europe 2001: 66). Bedenkt man darüber hinaus noch die durchschnittliche Länge einer HVA auf B1-Niveau, die bei ca. 3 Minuten liegt, so wird klar, dass eine einzige Aufgabe keinesfalls valide Aussagen über die Hörverstehenskompetenz der Lernenden zulässt. Eine einzige Aufgabe deckt weder das (default-)Konstrukt ab, noch kann sie das Konstrukt auch nur annähernd inhaltlich repräsentieren. Das ist auch der Grund, warum in die kriterienorientierte Qualifikationsprüfung der Standardisierten Reife- und Diplomprüfung (SRDP) in Österreich vier HVA aufgenommen wurden, von der jede zweimal vorgespielt wird, was nicht immer authentisch sein mag, letztendlich aber die Chancen des Verstehens erhöht und Störfaktoren minimiert (vgl. Grotjahn 2012: 80). Zudem wird durch die Zahl der vier Aufgaben auch eher der Repräsentationsgrad des Konstrukts erreicht. 


\section{Kompetenzorientierte HVA}

Wagner \& Werry (2015: 571) nennen fünf Eigenschaften, die Aufgaben im Fremdsprachenunterricht erfüllen müssen, um als „kompetenzorientiert" zu gelten und in der Folge einen positiven Washback auszulösen:

- Authentizität bzw. Wirklichkeitsnähe

- Situationseinbettung aus Schülerperspektive

- Aufforderungscharakter

- Relevanz und

- Diskriminierung.

Eine HVA erfolgreich bewältigen zu können bedeutet aus der Sicht der Autoren, die „relevante Information“ des Textes zu verstehen (ebd. 572). Diese fünf Eigenschaften sind auch Teil einer 10-Punkte-Charakteristik, die Wagner \& Werry (2015: 580-581) für ,kompetenzorientierte Klassenarbeiten (KOKA)“ bzw. informelle Tests aufstellen. Sie erläutern diese anschaulich, wenn auch minimalistisch, anhand eines einzigen MultipleChoice Items mit drei Optionen, das einen GeR-Deskriptor aus der Skala Ankündigungen, Durchsagen und Anweisungen verstehen umsetzt. Sie sagen des Weiteren, dass KOKA keineswegs (bezugs-)normorientiert ausgewertet bzw. bewertet werden müssen. KOKA sollen jene Aufgabenformate enthalten, die auch in standardisierten Tests zum Einsatz kommen ${ }^{5}$, um a) Lernende auf diese vorzubereiten und b) eine kontinuierliche, formative bzw. diagnostische Beurteilung zu ermöglichen, mit dem Ziel, konkretes und lernerInnenbezogenes Feedback geben zu können. KOKA sollen außerdem unterschiedliche Teilfertigkeiten im Auge behalten und den Gütekriterien Validität (Konstrukt, Inhalt) sowie (Auswertungs-)Objektivität gerecht werden. Vergleicht man diese Grundsätze (1-10) einer

\footnotetext{
5 Wir müssen davon ausgehen, dass Unterricht und Leistungsüberprüfung stark miteinander verbunden sind (vgl. Bérešová 2013: 180).
} 
„kompetenzorientierten Klassenarbeit““ (Wagner \& Werry 2015) mit dem Assessment Use Argument (AUA) für die Interpretation einer Leistungsbeurteilung nach Bachman \& Palmer (2010), so ergeben sich folgende Parallelen (s. Tabelle 1):

\begin{tabular}{|c|c|}
\hline $\begin{array}{c}\text { KOKA-Grundsätze nach Wagner \& } \\
\text { Werry (2015) }\end{array}$ & $\begin{array}{c}\text { Assessment Use Argument } \\
\text { nach Bachman \& Palmer } \\
\text { (2010) }\end{array}$ \\
\hline $\begin{array}{c}\text { standardbezogen (1) } \\
\text { konstrukt- bzw. inhaltsvalide (3) }\end{array}$ & aussagekräftig (meaningful) \\
\hline $\begin{array}{c}\text { objektiv, unabhängig von der Lehrkraft (6) } \\
\text { kriterien-, nicht normorientiert (8) } \\
\text { gezielte Diagnostik (9) }\end{array}$ & $\begin{array}{c}\text { verallgemeinerbar } \\
\text { (generalizable) }\end{array}$ \\
\hline $\begin{array}{c}\text { realistische /realitätsnahe Kontexte (4) } \\
\text { Voraussetzung grammatischer und le- } \\
\text { xikalischer Kompetenz (10) }\end{array}$ & relevant (relevant) \\
\hline $\begin{array}{c}\text { Relevanz und Aufforderungscharakter (5) } \\
\text { trennscharfe Items (7) }\end{array}$ & ausreichend (sufficient) \\
\hline Abdeckung mehrerer Fertigkeitsbereiche (2)
\end{tabular}

Tab. 1 Gegenüberstellung der KOKA-Grundsätze (Wagner \& Werry 2015) und dem Assessment Use Argument (Bachman \& Palmer 2010) (Eigene Darstellung A.D./W.St.)

Fassen wir zusammen: Um Entscheidungen über die Ergebnisse einer HVA machen zu können, die sich für den weiteren Unterricht bzw. die Einschätzung der Leistung als nützliche und aussagekräftige Entscheidungen erweisen, bedarf es konkreter Kenntnisse, wie eine Aufgabe erstellt, durchgeführt und wie Kompetenz und Performanz - Prozess oder Produkt der Hörleistung - interpretiert werden. Solch eine Aufgabe muss gewisse Standards erfüllen, die einem sprachlichen Kommunikationsmodell (wie jenem des GeR oder der entsprechenden (Rahmen-)Lehrpläne bzw. Curricula) entnommen sind. Diese Standards sind für die entsprechende Test- oder Prüfungsaufgabe bzw. die Klassenarbeit in Form von Testspezifikationen detailliert aufzuschlüsseln (vgl. Eberharter, Kremmel 
\& Zehentner 2018: 61-63). Testspezifikationen stellen einen „Bauplan“ dar, der das zu überprüfende Konstrukt für eine einzelne Klassenarbeit bzw. die gesamten Klassenarbeiten eines Schuljahres inhaltlich ausreichend repräsentiert.

Die objektive Interpretation der Ergebnisse von HVA kann einerseits mittels vorgegebener GeR-Kriterien, mithilfe geeigneter Testformate und durch denselben Durchführungsmodus für alle KandidatInnen und andererseits durch die Nutzung eines transparenten Bewertungsschlüssels, in dem festgelegt ist, wie viele Punkte pro Item vergeben werden, sichergestellt werden. Die kriterienbezogene Beurteilung lässt eine gezielte Diagnostik der Sprachkompetenz zu.

Die gezeigte Leistung ist für die außerschulische Verwendung in der Zielsprache verallgemeinerbar, wenn die Interaktion bei der Hörverständnisaufgabe dem Zweck einer möglichst authentischen bzw. realitätsnahen Rezeption entspricht. Nicht umsonst hebt der GeR (vgl. Europarat 2001: 74) die Empfehlung hervor, die BenutzerInnen sollten ,bedenken und, soweit sinnvoll, angeben, welches Spektrum an gesprochenem Input die Lernenden verstehen müssen, [...] mit welchen Absichten sie dem Input zuhören und auf welche Art und Weise sie zuhören werden“". Vor allem der Hinweis auf ,gesprochenen Input" im GeR legt nahe, nicht ausschließlich mit vorgelesenen, verschriftlichten Texten, sondern vor allem auch mit Hördateien und mündlichem Input zu arbeiten, weil die Erstellung und die Lösung der Aufgabe dadurch authentischer werden: ,, Scripted texts - speakers reading from prepared written texts - are the easiest texts to make, [but] they are generally the least appropriate" (vgl. https://tinyurl.com/y9w3cd8u).

Interpretationen sind ausreichend und relevant, wenn sie a) auf Ergebnissen basieren, denen ganzheitliches und tiefes Hörverstehen ( global, selektiv, detailliert und inferierend) vorausgeht (vgl. Wagner \& Werry 2015:

Anastasia Drackert und Katrin Bente Karl (Hg.), Didaktik der slawischen Sprachen

Beiträge zum 2. Arbeitskreis in Innsbruck (19.02.-20.02.2018)

(C) 2019 innsbruck university press, ISBN 978-3-903187-80-1, DOI 10.15203/3187-80-1 
582) und wenn sie b) helfen, Handlungen auszulösen, die adressatengerecht sind und lernstarke SchülerInnen von lernschwachen trennen.

Im Folgenden wird dargestellt, wie die Leistungsbeurteilungskompetenz von RussischlehrerInnen (pre-service und in-service) im Bereich Hörverstehen ausgebildet werden kann. Angesichts der Komplexität des Themas kann im Rahmen dieses Aufsatzes nicht auf alle o.g. theoretischen Aspekte eingegangen werden. Im Sinne einer didaktischen Reduktion sollen in der Aus- und Weiterbildung folgende für die Praxis wichtige drei Bereiche behandelt werden: a) die Schwierigkeit von HVA; b) Hörziele (das Konstrukt „Hörverstehen“) und c) Regeln zur Erstellung und Durchführung von HVA.

\section{Lehrszenario zur Ausbildung der Leistungsbeurteilungs- kompetenz im Bereich „Hörverstehen überprüfen““}

Das im Folgenden dargestellte „Lehrszenario in zwölf Schritten“ (s. Tabelle 2) wird von den Autoren sowohl in der universitären Ausbildung von Lehramtsstudierenden im Unterrichtsfach Russisch an der Ruhr-Universität Bochum und der Universität Innsbruck als auch bei zahlreichen Fortbildungen für Lehrkräfte angewendet und konsequent weiterentwickelt. Ungeachtet der zur Verfügung stehenden Zeit, gilt es in jedem Fall, die zu vermittelnden Inhalte nicht ausschließlich frontal und in Form reinen Inputs zu präsentieren, auch wenn Lehrende praktische Anweisungen in kurzer Form und als „fertige“ Handouts präferieren. Sondern es gilt, die Inhalte aufgaben- und handlungsorientiert erarbeiten zu lassen, um den erwartbaren Output der Lernenden in Bezug auf das zu überprüfende Konstrukt zu sehen (vgl. u.a. Fischer et al. 2011: 122). Vor allem sollen die Studierenden nachvollziehen können, was eine theoriegeleitete Unterrichtspraxis ausmacht. 


\begin{tabular}{|c|c|}
\hline Schritte & Lehrszenario \\
\hline 1 & Erstellung/Einreichung einer HVA auf B1-Niveau \\
\hline 2 & $\begin{array}{l}\text { Lektüre eines Fachtextes bzw. Aktivierung von Vorkenntnissen } \\
\text { mit HVA und Mindmap }\end{array}$ \\
\hline 3 & Besprechung der Mindmap \\
\hline 4 & $\begin{array}{l}\text { Überblick über wissenschaftliche Erkenntnisse zum Bewerten } \\
\text { von HVA (Bezug zur Fachliteratur) }\end{array}$ \\
\hline 5 & $\begin{array}{l}\text { Lösen verschiedener HVA und Besprechung der Aufgabenfor- } \\
\text { mate }\end{array}$ \\
\hline 6 & $\begin{array}{l}\text { Familiarisierung mit GeR-Deskriptoren „Hören“ (A2-B2 Ni- } \\
\text { veau) }\end{array}$ \\
\hline 7 & Adaption von Deskriptoren für einen spezifischen Task \\
\hline 8 & Text Mapping \\
\hline 9 & Richtlinien zur Erstellung von HVA \\
\hline 10 & Diskussion eines Best-Practice-Beispiels \\
\hline 11 & $\begin{array}{l}\text { Revision der im Voraus erstellten Aufgaben, ggf. Feedback } \\
\text { durch den/die LV- oder FortbildungsleiterIn }\end{array}$ \\
\hline 12 & $\begin{array}{l}\text { Komplettierung der Mindmap und Reflexion bzw. Evaluation } \\
\text { des Gelernten }\end{array}$ \\
\hline
\end{tabular}

Tab. 2 12-Schritte-Lehrszenario für Hörverstehensaufgaben erstellen lernen (Eigene Darstellung A.D. \& W.St.)

Vor der ersten Sitzung werden die Studierenden gebeten, zu zweit eine HVA zu einer Lektion bzw. zu einem Thema auf B1-Niveau zu erstellen und diese Aufgabe zu einer festgesetzten Deadline vor der ersten Sitzung einzureichen (Schritt 1). Als Vorbereitung sollen sie zudem entweder Kapitel 6 „Rezeptive Fertigkeiten überprüfen und bewerten“ (Stadler 2018: 69-86) aus der Einführung Testen und Bewerten fremdsprachlicher Kompetenzen oder Chapter 4 ,Assessing listening skills“ (Green 2017: 81-97) aus dem Handbook of Assessment for Language Teachers lesen und eine 
Mind-Map zum Thema „Testen des Hörverstehens“ erstellen (Schritt 2). ${ }^{6}$ Für eine Fortbildung werden die Teilnehmenden ersucht, eine bereits eingesetzte HVA auf dem Niveau B1 aus einer Klassenarbeit mitzubringen.

Die Präsenzveranstaltung für Studierende beginnt mit der Besprechung der Mind-Map (Schritt 3) bzw. setzt für die TeilhnehmerInnen einer Fortbildung mit dem Erstellen einer solchen zum Thema „Testen des Hörverstehens" ein. Dabei werden die Vorkenntnisse der TeilnehmerInnen aktiviert, festgehalten und mit ihnen diskutiert. Im Anschluss daran kann eine kurze Einführung zur Theorie folgen (Schritt 4), in der die Besonderheiten der Fertigkeit Hörverstehen und die Hörziele besprochen werden (siehe Abschnitt 2 dieses Beitrags: Theoretischer Hintergrund).

Nach der Besprechung der Theorie findet eine handlungsorientierte Auseinandersetzung mit der Schwierigkeit von HVA und Aufgabenformaten statt: Studierende ${ }^{7}$ lösen einzelne HVA in unterschiedlichen Aufgabenformaten zu demselben Hörtext. Als Beispiel dient die Hördatei «Учёба в чистом поле» (Cvetova 2013) und die dazu erstellten Aufgaben (siehe Anhang 1). Nach dem Lösen der Aufgabe bewerten die Studierenden den Schwierigkeitsgrad der jeweiligen Aufgabe auf einer Skala von 1 (sehr einfach) bis 10 (sehr schwierig). Mit dieser Aktivität wird evidenzbasiert veranschaulicht, dass der Schwierigkeitsgrad der Aufgabe trotz desselben Inputtexts aufgrund der unterschiedlichen Aufgabenformate variieren kann. Aufbauend auf dieser gewonnenen Erfahrung folgt anschließend eine Auseinandersetzung mit den Vor- und Nachteilen der einzelnen Aufgabenformate (Kurzantworten, Erstellen einer Zusammenfassung, Vervollständigung von Sätzen bzw. Tabellen, Zuordnung, Richtig/Falsch (ohne Begründung), Richtig/Falsch (mit Begründung), Multiple Choice,

\footnotetext{
${ }^{6}$ Diese Mind-Map kann auch mit einem Online-Tool wie Mindmeister, Mapmyself oder Wisemapping erstellt werden. Ein mit Mindmeister erstelltes Beispiel findet sich z.B. unter https://tinyurl.com/y9kcm79s.

${ }^{7}$ Im Folgenden ist der Einfachheit halber von Studierenden die Rede. Es können aber auch TeilnehmerInnen an Fortbildungen, Referendare, FachleiterInnen etc. gemeint sein.
} 
Lückendiktat) sowie mit ihrem Einfluss auf das zu messende Konstrukt (Schritt 5).

Da Konstruktvalidität als Charakteristikum eines guten Tests von den Russischlehrkräften nicht so oft genannt wurde (vgl. die Untersuchung von Drackert \& Stadler 2017), diese für die Interpretation der Schülerleistungen allerdings maßgeblich ist, wird im nächsten Schritt anhand ausgewählter GeR-Deskriptoren und der Hördatei «Спортсекции» (B1) (VRÖ) mithilfe der Methode des Text Mapping auf das Konstrukt des Hörverstehens näher eingegangen. Die Studierenden erhalten eine Liste mit Deskriptoren (zwischen zehn und zwölf an der Zahl), in der sie (je nach der zur Verfügung stehenden Zeit) entweder jeden Deskriptor der entsprechenden Kompetenzniveaustufe zuordnen oder nur jene Deskriptoren markieren, die dem Niveau der Kompetenzstufe B1 entsprechen (Schritt 6). Ein Beispiel für eine Liste (mit Lösung) findet sich in Anhang 2. Es ist empfehlenswert, in die Liste der Deskriptoren ca. die Hälfte aus dem Niveau B1 aufzunehmen, da ja genau mit dieser Stufe ein Vertrautwerden erreicht werden soll. Der Rest der „Kann-Beschreibungen“ sollte aus den angrenzenden Levels A2 und B2 stammen. Dieser Prozess der Familiarisierung (vgl. Council of Europe 2009) ist notwendig, um im Folgenden einschätzen zu können, ob der Text «Спортсекции» für die Niveaustufe B1 geeignet ist. An diesen Prozess der Zuordnung (Deskriptor $\rightarrow$ Niveaustufe) schließt eine Diskussion an, in der die Studierenden argumentieren, warum sie sich für das eine oder andere Niveau entschieden haben. Dabei sollen sie eine Tendenz in der eigenen Zuordnung erkennen, d.h. sie sollen herausfinden, ob sie eher zu einer Über- oder einer Unterschätzung der beschriebenen Leistung neigen. Es geht also nicht darum, wer die meisten richtigen Zuordnungen erzielt.

Anschließend wird den Studierenden die Hördatei «Спортсекции» zweimal vorgespielt und sie werden gebeten, die identifizierten B1Deskriptoren aus der Liste so zu adaptieren, dass sie den erlebten und notwendigen Verstehensprozessen entsprechen. Damit wird gewährleistet,

Anastasia Drackert und Katrin Bente Karl (Hg.), Didaktik der slawischen Sprachen

Beiträge zum 2. Arbeitskreis in Innsbruck (19.02.-20.02.2018)

(C) 2019 innsbruck university press, ISBN 978-3-903187-80-1, DOI 10.15203/3187-80-1 
dass die Auswahl einer Hördatei mit den Deskriptoren einer bestimmten Kompetenzniveaustufe korrespondiert (Schritt 7). Um sicherzustellen, dass sinnerfassendes Hören nicht das Ergebnis der subjektiven Einzeleinschätzung eines Testerstellers ist, raten wir an, vor der Entwicklung der Testaufgabe ein Text Mapping zu machen (Schritt 8), das z.B. „Kernaussagen identifiziert, die als Grundlage bei der Itemerstellung herangezogen werden“ (Eberharter, Kremmel \& Zehentner 2018: 63 ff). Auf Niveau B1 empfiehlt sich sowohl ein Text Mapping für das Identifizieren von Kernaussagen (main points) als auch ein Identifizieren von Einzelinformationen (specific details). ${ }^{8}$

Im nächsten Schritt folgt ein Input mit den Regeln zur Erstellung und Durchführung von HVA, die in Form einer Checkliste (siehe Anhang 3) zusammengefasst sind (Schritt 9). Eine gründliche Diskussion dieser Checkliste wird vorausgesetzt. Als zehnter Schritt wird den Studierenden ein auf der Basis dieses Lehrszenarios erstelltes Best practice-Beispiel präsentiert (s. Anhang 4). Anhand der Checkliste können sie diese Aufgaben zu zweit oder in kleinen Gruppen evaluieren und, wenn aus ihrer Sicht notwendig, verbessern und gleichzeitig die Gütekriterien einer HVA besprechen. Bei Bedarf kommt entsprechendes Feedback von dem/der LehrveranstaltungsleiterIn (Schritt 11).

Am Ende der Veranstaltung findet eine Reflexion und Evaluation des Gelernten statt (Schritt 12). Als Erstes werden die Studierenden gebeten, die Mind-Maps in einer zusätzlichen Farbe zu ergänzen und so ihren Lernertrag deutlich $\mathrm{zu}$ visualisieren. Bei vorhandenen zeitlichen Kapazitäten kann anschließend noch im Plenum diskutiert werden, wie die gewonnenen (Er-)Kenntnisse z.B. auf das Überprüfen und Bewerten von Lesekompetenz übertragen werden können.

\footnotetext{
${ }^{8}$ Genauere Anleitungen für das Durchführen eines Text Mapping finden sich z.B. in Green (2017), Kapitel 3, S. 57 ff.
} 


\section{Fazit}

Es ist uns bewusst, dass solch eine exemplarische Behandlung nicht eine ganze Lehrveranstaltung oder einen Fortbildungszyklus ersetzen kann, doch stellt das Lehrszenario in zwölf Schritten (s. Tabelle 2 in Abschnitt 5) eine Hilfestellung dar, wie die Sprachbewertungskompetenz (language assessment literacy) von Lehrkräften bewusst entwickelt und gefördert werden kann.

Bei der Überprüfung und Bewertung der produktiven Fertigkeiten ließe sich analog das Hauptaugenmerk im Lehrszenario entweder auf die Fertigkeit Schreiben oder Sprechen richten, wobei ein Schwerpunkt auf der Arbeit mit Bewertungsrastern liegen müsste.

Wichtig scheint uns auch, in der Ausbildung die Studierenden handlungsorientiert agieren zu lassen und ihnen nicht nur Literaturempfehlungen zu geben oder Checklisten zur kritiklosen und passiven Übernahme vorzulegen. Schließlich sollen keine „Testproduktionsroboter“ ausgebildet werden. Nicht nur als LehrerIn, sondern auch als PrüferIn und BewerterIn ist kritisches Denken erforderlich: Die eigenen Handlungen - sei es das Bestimmen des Konstrukts, die Auswahl oder das Erstellen von Aufgaben für die Praxis und die Interpretation der Ergebnisse - sollten immer mit Bezug zur Theorie hinterfragt werden.

Eine bessere Verzahnung der Phasen in der pre-service Ausbildung mit jenen in in-service-Veranstaltungen liegt nahe und kann sich an folgenden Fragen orientieren: Was ist in den fachdidaktischen Kursen an der Universität zu vermitteln? Was soll im Referendariat, in den Fach- und Schulpraktika bzw. in der LehrerInnenfortbildung ergänzt werden? 
Das präsentierte Lehrszenario scheint uns übrigens auch auf die Ausbildung von Lehrkräften in anderen fremdsprachlichen Unterrichtsfächern übertragbar.

\section{Literatur}

Anderson, A. \& Lynch, T. (1988). Listening. Oxford: Oxford University Press.

Anisimova = Анисимова, И.Н. (2015). Об аудировании в процессе обучения русскому языку иностранных студентов экономического факультета. Научно-методический электронный журнал «Конщепт», 24, 11-15.

Averko-Antonovič = Аверко-Антонович, Е.В. (2013). Аудирование в изучении русского языка иностранными студентами. Вестник Казанского технологического университета, 16 (15).

Bachman, L \& Palmer, S. (2010). Language Assessment in Practice. Oxford: Oxford University Press.

Balas, A., Kaltseis, M. \& Schmidt, Chr. (2017). Eine Analyse der Aufgabenauthentizität in drei verschiedenen Ausgaben des Russischlehrwerks Dialog 1. Unveröffentlichte Seminararbeit. Leopold-Franzens-Universität Innsbruck und Humboldt-Universität zu Berlin.

Bauer, F. \& Kolesnik-Eigentler, M. (2013). Auf dem Weg zur neuen Reifeprüfung Russisch. Aufgaben und Übungsbeispiele zur Vorbereitung auf die neue Matura. Eisenstadt: Weber.

Bauer, S. (1986). Zu Komponentenübungen im verstehenden Hören im Russischunterricht der zehnklassigen allgemeinbildenden polytechnischen Oberschule der DDR (Diss. A). Dresden.

Beréšová, J. (2013). Teaching and Testing are Interrelated. But to What Extent? Verfügbar unter: https://tinyurl.com/y99kub8k [24.09.2018]. 
Birzer, S. \& Steinbach, A. (2015). Hörverstehen im Russischunterricht. Verfügbar unter: https://tinyurl.com/ybq8v5j7 [17.10.2018].

Boge, C. (2012). Hörverstehen im Englischunterricht von der Primarstufe bis zum Abitur: Kompetenzerwartungen, Problemanzeigen und fachdidaktische Lösungsansätze. Peer reviewed paper delivered at the GMF Conference (Bundeskongress Gesamtverband Moderne Fremdsprachen) held at the University of Duisburg-Essen, North Rhine-Westphalia, Germany, September 13 to 15, 2012.

Buck, G. (2001). Assessing listening. Cambridge: Cambridge University Press.

Chastain, K. (1979). Testing Listening Comprehension Tests. Tesol Quarterly, 81-88.

Conti, G. (2015). Listening - the often 'mis-taught' skill. Part one: the issues undermining aural skills instruction. The Language Gym. Verfügbar unter: https://tinyurl.com/yax2wvxx [24.09.2018].

CEFR = Council of Europe. (2001). Common European Framework of Reference for Languages: Learning, teaching, assessment. Cambridge, UK: Cambridge University Press.

Council of Europe (2009). Relating Language Examinations to the Common European Framework of Reference for Languages: Learning, Teaching, Assessment (CEFR): A Manual. Strasbourg: Council of Europe Language Policy Division.

Council of Europe (2018). Common European Framework of Reference for Languages: Learning, Teaching, Assessment. Companion Volume with New Descriptors. Verfügbar unter: https://tinyurl.com/ybjrszaa, für die französischsprachige Fassung: https://tinyurl.com/y3sf6hwg [18.06.2018].

Cvetova = Цветова, H.Е. (2013). 112 тестов по русскому языку как иностранному. Уровни А1-В1. СПб: Златоуст.

Drackert, A. \& Stadler, W. (2017). Leistungsbeurteilungskompetenz von Russischlehrkräften in Deutschland, Österreich, der Schweiz und

Anastasia Drackert und Katrin Bente Karl (Hg.), Didaktik der slawischen Sprachen

Beiträge zum 2. Arbeitskreis in Innsbruck (19.02.-20.02.2018)

(C) 2019 innsbruck university press, ISBN 978-3-903187-80-1, DOI 10.15203/3187-80-1 
Südtirol (DACHS): Zwischen Status Quo und aktuellen Bedürfnissen. Zeitschrift für Fremdsprachenforschung (ZFF), 28 (2), 233258.

Eberharter, K., Kremmel, B. \& Zehentner, M. (2018). Die Erstellung von Testaufgaben: Der Testentwicklungszyklus. In B. Hinger \& W. Stadler (Hrsg.), Testen und Bewerten fremdsprachlicher Kompetenzen. Eine Einführung (57-68). Tübingen: Narr Studienbücher.

Field, J. (2011). Cognitive Validity. In L. Taylor (Hrsg.) Examining Speaking: Research and Practice in Assessing Second Language Speaking. (65-111). Cambridge: Cambridge University Press.

Fischer, J., Chouissa, C., Dugovičová, St. \& Virkkunen-Fullenwider, A. (2013). Guidelines for task-based university language testing. Verfügbar unter: https://tinyurl.com/yanvx7zz [24.09.2018].

Fischer, Y. (2015). Das Hörverstehen mit einem Raplied fördern. PRAXIS Fremdsprachenunterricht Russisch, 3, 7-9.

Fitzer, Т. (2014). «Показ мод» Hörverstehen entwickeln und spielerisch überprüfen. PRAXIS Fremdsprachenunterricht Russisch, 6, 7-9.

$\mathrm{GeR}=$ Europarat (2001): Gemeinsamer europäischer Referenzrahmen für Sprachen: Lernen, lehren, beurteilen. Berlin u. a.: Langenscheidt.

Green, A. (2018). Assessing Listening Skills. In Tsagari, D., Vogt, K., Fröhlich, V. et al. (Hrsg.), Handbook of Assessment for Language Teachers. www.taleproject.eu, 81-97.

Green, R. (2017). Designing Listening Tests. A Practical Approach. London: Palgrave Macmillan.

Grotjahn, R. (2012). Hörverstehen: Konstrukt und Messung. FLuLFremdsprachen Lehren und Lernen, 41 (1), 72-86.

Guan, X. \& Jin, Y. (2010). Interactive Listening: Construct Definition and Operationalization in Tests of English as a Foreign Language. In Chinese Journal of Applied Linguistics (Foreign Language Teaching \& Research Press), 33 (6), 16-39.

British Council. Guidelines for Writers of Listening Tests. Verfügbar unter: https://tinyurl.com/y7rqrnq3 [24.09.2018].

Anastasia Drackert und Katrin Bente Karl (Hg.), Didaktik der slawischen Sprachen

Beiträge zum 2. Arbeitskreis in Innsbruck (19.02.-20.02.2018)

(C) 2019 innsbruck university press, ISBN 978-3-903187-80-1, DOI 10.15203/3187-80-1 
Heyer, Ch. (2010). П(р)ослушайте и ..., Hörverstehen im Anfangsunterricht. PRAXIS Fremdsprachenunterricht Russisch, 5, 4-6.

Heyer, Ch. (2014). Hörverstehen, Hör-Seh-Verstehen. In A. Bergmann (Hrsg.), Fachdidaktik Russisch. Eine Einführung (108-120). Tübingen: Narr Studienbücher.

Iskold, L. (2008). Research-Based Listening Tasks for Video Comprehension.

Khalifa, H. \& Weir, C. (2009). Examining Reading: Research and Practice in Assessing Second Language Learning. Cambridge: Cambridge University Press.

Kranert, M. (2013). Korrigieren, Prüfen und Testen im Fach Deutsch als Fremdsprache. Ein kurzer Leitfaden. Verfügbar unter: https://tinyurl.com/yak62rb3 [21.09.2017].

Masalimova, A., Porchesku, G. \& Liakhnovitch, T. (2014). Linguistic Foundation of Foreign Language Listening Comprehension. International Electronic Journal of Mathematics Education, 11 (1), 123131.

Nguyen, H. \& Abbott, M. (2016). Promoting Process-Oriented Listening. Instruction in the ESL Classroom. TESL Canada Journal, 34 (11), $72-86$.

Nold, G. \& Rossa, H. (2007). Hörverstehen. In B. Beck \& E. Klieme (Hrsg.), Sprachliche Kompetenzen. Konzepte und Messung. DESIStudie (Deutsch Englisch Schülerleistungen International) (178196). Weinheim u.a.: Beltz.

Nunan, D. (1997). Listening In Language Learning. Verfügbar unter: https://tinyurl.com/y9aa2ztd [24.09.2018].

Osada, N. (2004). Listening Comprehension Research: A Brief Review of the Past Thirty Years. Dialogue, 3 (1), 53-66.

Ott, J. (1995). Hören - Verstehen - Begreifen: eine interkulturelle Analyse einer HV-Sequenz. Die Neueren Sprachen (DNS), 94 (5), 514-532.

Porsch, R., Grotjahn, R. \& Tesch, B. (2010). Hörverstehen und Hör-Sehverstehen in der Fremdsprache - unterschiedliche Konstrukte? Zeitschrift für Fremdsprachenforschung, 21 (2), 143-189.

Anastasia Drackert und Katrin Bente Karl (Hg.), Didaktik der slawischen Sprachen

Beiträge zum 2. Arbeitskreis in Innsbruck (19.02.-20.02.2018)

(C) 2019 innsbruck university press, ISBN 978-3-903187-80-1, DOI 10.15203/3187-80-1 
Sherrow R. (1971). Suggestions for Teaching Listening Comprehension Skills. The French Review, 44 (4), 738-742.

Stadler, W. (2018). Rezeptive Fertigkeiten überprüfen und bewerten, In B. Hinger \& W. Stadler (Hrsg.) Testen und Bewerten fremdsprachlicher Kompetenzen. Eine Einführung (69-86). Tübingen: Narr Studienbücher.

Thaler, E. (2009). Hörverstehen. PRAXIS Fremdsprachenunterricht, 5, 55. Thompson, I. \& Rubin, J. (1996). Can Strategy Instruction Improve Listening Comprehension? Foreign Language Annals, 29 (3), 331-342.

Vandergrift, L. \& Goh, C. (2009). Teaching and Testing Listening Comprehension. In M. Long \& C. Doughty (Hrsg.), The Handbook of Language Teaching (395-411). Chichester: Wiley-Blackwell.

Vandergrift, L. (1999). Facilitating Second Language Listening Comprehension: Acquiring Successful Strategies. ELT Journal Volume, 53 (3), 168-176.

Volosova = Волосова, М.В. (2017). Роль аудирования в процессе обучения говорению на русском языке как иностранном. Педагогический журнал, 7 (1А), 208-216.

Vorabhinweise zum Abitur 2018. Allgemein bildende Fächer. Eine Handreichung für Lehrerinnen und Lehrer. Mecklenburg Vorpommern. Institut für Qualitätsentwicklung. Verfügbar unter: https://tinyurl.com/y9docnsu [24.09.2018] 1-4.

Wagner, E. \& Werry, H. (2015). Kompetenzorientierter Fremdsprachenunterricht: neue Formen der Leistungsmessung in Klassenarbeiten und ihre Wirkung auf den Unterricht. In J. Böcker \& A. Stauch (Hrsg.) Konzepte aus der Sprachlehrforschung - Impulse für die Praxis. Festschrift für Karin Kleppin. (569-592). Frankfurt am Main: Peter Lang.

Walker, N. (2014). Listening: The Most Difficult Skill to Teach. Encuentro, 23, 167-175. 


\section{Anhang 1}

\section{Höraufgaben zum Нörtext «Учёба в чистом поле» in unterschiedlichen Aufgabenformaten (erstellt an der Ruhr-Universität Bochum) ${ }^{9}$}

\section{1) Kurzantworten zu den Fragen}

Прослушайте текст и ответьте на вопросы.

(1) Где находится летняя школа?

(2) Откуда приезжают участники?

(3) Кто читает лекции в летней школе?

(4) Кто такой Пётр Александрович Ореховский?

\section{2) Erstellen einer Zusammenfassung}

Прослушайте текст,

(1) определите его тему и

(2) передайте его главное содержание.

\section{3) Vervollständigung von Sätzen, Tabellen}

Дополните предложения.

(1) Летняя школа называется

и находится в

(2) Участники приезжают из

и проводят здесь

(3) Люди со стороны думают, что летняя школа не

(4) Один участник встретил учёного

9 Diese Aufgaben dienen nicht als Best-Practice-Beispiele, sondern zur Veranschaulichung unterschiedlicher Testformate. Auf die problematischen Stellen bei den einzelnen Aufgaben kann eingegangen werden, nachdem die Richtlinien zur Erstellung von HVA vorgestellt wurden.

Anastasia Drackert und Katrin Bente Karl (Hg.), Didaktik der slawischen Sprachen

Beiträge zum 2. Arbeitskreis in Innsbruck (19.02.-20.02.2018)

(C) 2019 innsbruck university press, ISBN 978-3-903187-80-1, DOI 10.15203/3187-80-1 


\section{4) Zuordnung}

Что думают участники о летней школе? Соедините ... .

Один вариант здесь лишний.

(1) Ян

(первый участник)

(2) Анастасия

(второй участник)

(3) Надежда

(третий участник)

(4) Максим

(четвёртый участник)
А ___ Я познакомился с учёным высокого уровня.

В ___ У меня появилось желание, самостоятельно учиться.

С__ Я нашёл здесь новых друзей, а школа мне как семья.

D___ Я только несколько дней принимала участие в школе.

Е___ Я очень старался и много что узнал.

\section{5) Richtig/Falsch ohne Begründung}

Правильно или неправильно?

да нет

(1) Летняя школа не принимает школьников.

(2) Летняя школа разрешает также студентам прочитать лекции.

(3) Летняя школа работает круглый год.

(4) Летняя школа - безмятежный отдых.

\section{6) Richtig/Falsch mit Begründung}

Правильно или неправильно? Исправьте неправильные ответы.

(1) В мастер-классе участвуют опытные специалисты. $\quad$ д $\square \quad$ нет $\square$ потому что

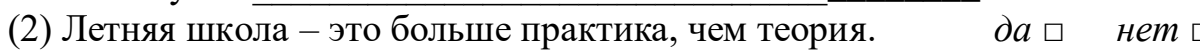
потому что

(3) Пётр Александрович Ореховский - директор летней школы.

потому что

(4) Ян (первый участник) встретил профессора нескольких крупных

российских вузов. $\quad \partial a$ нет $\square$ потому что 


\section{7) Multiple-Choice}

Прослушайте текст и выгерите правильный вариант ответа.

(1) Летняя школа открыта ...

A $\square$ круглый год

$\mathrm{B} \square$ на каникулах

$\mathrm{C} \square$ четыре месяца в году
(2) Опытные специалисты ...

$\mathrm{A} \square$ являются участниками мастеркласса

В $\square$ выбирают участников мастеркласса

$\mathrm{C} \square$ являются опытными мастерами

в классе

(4) Пётр Александрович

Ореховский - это ...

А $\square$ экономист и директор

российского вуза

$\mathrm{B} \square$ профессор в летней школе

$\mathrm{C} \square$ учёный, специалист высокого уровня

\section{8) Lückendiktat}

Слушайте текст и заполняйте пропуски.

\section{Учёба в чистом поле}

В Тверской области уже несколько лет подряд работает летняя школа «Исследователь». Это большой образовательный проект, в котором принимают участие школьники, студенты и преподаватели из разных городов России и из-за рубежа. Они приезжают сюда на месяц во время своих законных каникул и отпусков.

Здесь все учат и учатся. Прослушав лекцию, студенты сами становятся преподавателями на следующей паре. А опытные специалисты, прочитав курс лекций, оказываются в числе участников мастер-класса. Летняя школа - это не столько теория, сколько практика.

У человека со стороны такого рода проекты вызывают закономерный вопрос: что заставляет перегруженных учёбой и работой людей отказаться от безмятежного отдыха? Можно долго рассуждать на эту тему. Но лучше почитать выдержки из дневников участников школы. 
«Здесь легко дышать. Среди своих вообще дышится легко. Легко живётся, учится, спорится, да что угодно! Школа дает стимул к самообразованию. Я видел, как уже состоявшийся репортёр записывал в блокнот названия фильмов и книжек, о которых услышал именно здесь» (Ян Тяжлов, Белгород).

«В лагерь приехал новый гость, и нас попросили показать ему дорогу к реке. Пока шли, знакомились. Пётр Александрович Ореховский - профессор нескольких крупных российских вузов, экономист, учёный. В моём городе сложно увидеть специалиста такого уровня, тем более так запросто поговорить. Личности такого масштаба встречаются на занятиях летней школы очень часто» (Анастасия Якорева, Саратов).

«Пока ехала домой, думала, как это я за обычные четыре дня столько всего успела. Может, дело в том, что в школе нет посторонних и у каждого свои обязанности? В следующем году хочу приехать на всю смену» (Надежда Боровик, Москва).

«Перелистывал конспекты, скопившиеся, пока учился. Почти вся тетрадь исписана. Много мыслей, много встреч, много информации. Вернусь домой - долго ещё буду всё это переваривать» (Максим Олейников, Ростов-наДону). 


\section{Musterlösung Aufgabenformate}

1) Kurzantworten zu den Fragen

(1) В Тверской области; (2) из разных городов России и из-за рубежа;

(3) студенты и опытные специалисты; (4) профессор нескольких российских вузов, экономист, учёный;

3) Vervollständigung von Sätzen, Tabellen

(1) исследователь; в Тверской области; (2) из разных городов России и изза рубежа; один месяц; (3) безмятежный отдых; (4) экономиста;

4) Zuordnung

Von oben nach unten in der Reihenfolge: (2); (1); (3); (4)

Überflüssige Aussage: Я нашёл здесь новых друзей, а школа мне как семья.

5) Richtig/Falsch ohne Begründung

(1) нет (2) да (3) нет (4) нет

6) Richtig/Falsch mit Begründung

(1) да; (2) да; (3) нет, потому что он профессор нескольких вузов и приехал в летнюю школу в гости; (4) нет, например, потому что его встретила Анастасия;

7) Multiple-Choice

(1) В на законных каникулах; (2) А являются участниками мастер-класса; (3) C не безмятежный отдых; (4) С учёный, специалист высокого уровня; 


\title{
Anhang 2
}

\section{Liste mit Hördeskriptoren (erstellt an der Universität Innsbruck)}

\author{
Familiarisierung Hörverstehen (mit Lösung)
}

\section{H 1 (B1)}

Ich kann normalerweise den wesentlichen Punkten einer längeren Diskussion um mich herum folgen.

\section{H 2 (A2)}

Ich kann einfache Erklärungen (z.B. wie man zu Fuß oder mit öffentlichen Verkehrsmitteln an einen bestimmten Ort gelangt) verstehen.

\section{H 3 (B2)}

Ich kann genau verstehen, was mir in gesprochener Standardsprache gesagt wird, selbst bei Hintergrundgeräuschen.

\section{H 4 (A2)}

Ich kann genug verstehen, um konkreten Bedürfnissen im alltäglichen Leben gerecht zu werden.

\section{H 5 (A2)}

Ich kann kurzen, langsam und deutlich gesprochenen Aufnahmen über vorhersehbare, alltägliche Dinge die wesentliche Information entnehmen.

\section{H 6 (B1)}

Ich kann einem Vortrag oder einem Gespräch eines mir vertrauten Fachgebiets folgen, wenn der Vortrag einfach und klar strukturiert ist.

\section{H 7 (B2)}

Ich kann die meisten Reportagen in Standardsprache verstehen und dabei die

Stimmung, den Tonfall usw. des Sprechers /der Sprecherin heraushören.

\section{H 8 (B1)}

Ich kann die Hauptpunkte verstehen, wenn in deutlich artikulierter Standardsprache über vertraute Dinge gesprochen wird, denen man normalerweise bei der Arbeit, in der Ausbildung oder der Freizeit begegnet. 


\section{H 9 (B2)}

Ich kann die Hauptaussagen komplexer Redebeiträge sowohl zu konkreten als auch zu abstrakten Themen, einschließlich technischer Diskussionen innerhalb meines Fachgebiets verstehen.

\section{H 10 (B1)}

Ich kann Gesprächen über geläufige, regelmäßig vorkommende Ereignisse die Hauptaussagen entnehmen, wenn deutlich und in Standardsprache gesprochen wird.

\section{H 11 (B1)}

Ich kann in Radionachrichten und in einfacheren Tonaufnahmen über vertraute Themen die Hauptpunkte verstehen, wenn relativ langsam und deutlich gesprochen wird.

\section{H 12 (B2)}

Ich kann den wesentlichen Punkten von Vorträgen, Gesprächen und Berichten folgen. 


\section{Anhang 3}

\section{Checkliste zur Erstellung und Durchführung von HVA}

Checkliste (1)

Aufgabenerstellung

Die Texte sollen möglichst authentisch sein.

Ein spezielles Hintergrundwissen soll nicht erforderlich sein.

Antworten dürfen nicht möglich sein, ohne den Text zu hören.

Die Themen sollen dem Alter der Adressatengruppe entsprechen.

Texte, Sprechgeschwindigkeit und Länge müssen dem Schwierigkeitsgrad der Kompetenzniveaustufe gerecht werden.

$\square$ Innerhalb der Aufgabe darf das Testformat nicht gewechselt werden.

Eine Aufgabe umfasst mindestens 5 Items.

$\square$ Die Items sind gleichmäßig über den Text verteilt.

$\square$ Die Reihenfolge der Items ist chronologisch.

$\square$ Die Items sind voneinander unabhängig.

$\square$ Das erste Item dient als Beispiel.

$\square$ Die sprachliche Formulierung der Items liegt unter dem getesteten Schwierigkeitsgrad.

Die Aufgabe weist einen konkreten Lösungsschlüssel auf

Das Aufgabenformat wurde im Unterricht geübt.

Das Layout ist übersichtlich gestaltet.

Mindestens zwei verschiedene Hörverständnisziele werden überprüft.

$\square$ Für HerkunftssprecherInnen kann ein anderes Aufgabenformat gewählt werden oder zusätzliche Items erstellt werden.

Checkliste (2)

\section{Erstellung von MC-Items}

$\square$ Erstellen Sie MC-Items mit nur einer korrekten Lösung.

$\square$ Ein Hörverstehens-MC-Item sollte nicht mehr als drei Optionen (Wahlmöglichkeiten) haben, damit die Teilnehmenden nicht zu viel lesen müssen.

$\square$ Die Optionen sollten annähernd gleich lang sein.

Die Distraktoren (die nicht-korrekten Optionen) sollten plausibel sein. 
$\square$ Die Optionen sollten gut und leicht verständlich sein, z.B. keine doppelte Verneinung enthalten.

$\square$ Wenn Sie mehrere MC-Aufgaben hintereinander einsetzen, achten Sie darauf, dass die korrekte Lösung jeweils an einer anderen Position steht.

\section{Durchführung einer HVA}

Achten Sie auf die Qualität der Datei bzw. der Lautstärke.

$\square$ Verteilen Sie die Aufgabe(n) vor dem Hören und geben Sie den KandidatInnen Zeit, diese zu verstehen.

$\square$ Spielen Sie die Texte in der Regel zwei Mal ab.

$\square$ Lesen Sie den Text nicht vor.

$\square$ Geben Sie Zeit zum Niederschreiben der Aufgaben. 


\section{Anhang 4}

Прослушайте речь завуча (der stellvertretenden Schuldirektorin) школь о спортивных секииях. Перед началом прослушивания у Вас будет одна минута, чтобы прочитать вопросы. Затем Вы прослушаете запись 2 раза. Выберите правильный вариант ответа. Пример (0) уже выполнен. После второго прослушивания у Вас будет одна минута, чтобы проверить правильность Ваших ответов.

\section{СПОРТИВНЫЕ СЕКЦИИ}

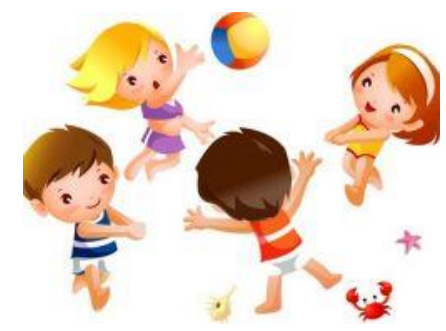

https://zelsport.ru

\section{0. К кому обращается Тамара Николаевна Литвиненко?}

A $\square$ ко всем школьникам

В $\square$ к Николаю Волкову

$\mathrm{C} \square$ к родителям

\section{1 Какая профессия у Николая} Волкова?
А $\square$ тренер баскетбольной команды
$\mathrm{B} \square$ профессиональный спортсмен
$\mathrm{C} \square$ руководитель спортивного комплекса

\section{2 Чем интересуются 20\% учашихся школы?}
A $\square$ бадминтоном
$\mathrm{B} \square$ баскетболом
$\mathrm{C} \square$ бодибилдингом 
3 Почему три процента учащихся не хотят заниматься спортом в школе?
A $\square$ у них нет времени
$\mathrm{A} \square 15 \%$
$\mathrm{B} \square$ они уже бегают по утрам
$\mathrm{B} \square 20 \%$
$\mathrm{C} \square$ они не любят спорт
$\mathrm{C} \square 25 \%$

\section{5 Кто будет оплачивать} основную часть стоимости секции?
А $\square$ родители
$\mathrm{B} \square$ школа
$\mathrm{C} \square$ Николай Волков

7 Что состоится в мае?
A $\square$ соревнования
$\mathrm{B} \square$ поездка на Байкал
$\mathrm{C} \square$ каникулы

\section{4 Сколько процентов учащихся} интересуются хоккеем?

\section{6 Когда начнутся тренировки?}

A $\square$ в марте

$\mathrm{B} \square$ в октябре

$\mathrm{C} \square$ в сентябре

8 Где будет проходить летний спортивный лагерь?
A $\square$ в горах
$\mathrm{B} \square$ на озере Байкал
$\mathrm{C} \square$ на реке

Набранные баллы: 18

Lösung

\begin{tabular}{|c|c|c|c|c|c|c|c|c|}
\hline 0 & $\mathbf{1}$ & $\mathbf{2}$ & $\mathbf{3}$ & $\mathbf{4}$ & $\mathbf{5}$ & $\mathbf{6}$ & $\mathbf{7}$ & $\mathbf{8}$ \\
\hline $\boldsymbol{A}$ & $\mathbf{C}$ & B & B & C & C & B & A & B \\
\hline
\end{tabular}


